\title{
Learning and motivation: Computer simulations of the Rescorla-Wagner, Solomon-Corbit, and Schull models
}

\author{
NIGEL W. BOND and DAVID A. T. SIDDLE \\ Macquarie University, Sydney, New South Wales, Australia
}

\begin{abstract}
A suite of programs is described for simulating the Rescorla-Wagner (1972) model of associative learning, the Solomon and Corbit (1974) opponent-process theory, and Schull's (1979) extension of the latter. The programs are written for the Commodore 64 computer.
\end{abstract}

Mathematical models of learning and motivation lend themselves to simulation on the currently available microcomputers. Examples include the Rescorla-Wagner (1972) model of associative learning, the opponentprocess theory of Solomon and Corbit (1974), and its extension by Schull (1979). The present paper describes a suite of programs that enables students to work through simulations of these models.

The programs have been written for the Commodore 64 microcomputer, equipped with the Simons's Extended BASIC cartridge. Because they make extensive use of color, the high-resolution graphics mode, and the realtime clock of the Commodore 64, the programs are unlikely to run on other systems without extensive revision. A graphics printer, such as the Commodore MPS-803, enables the user to produce a hard copy of much of the output of the simulations.

There are two programs, one for the Rescorla-Wagner model and one for the Solomon-Corbit and Schull models. Each is menu driven, and students control their rate of progress through manipulation of the function keys. Errors are easily corrected and, where appropriate, error checking prevents the keying in of errors that are likely to crash the program.

\section{RESCORLA-WAGNER SIMULATION}

The Rescorla-Wagner model is a linear model of associative learning (Rescorla \& Wagner, 1972). The change in associative strength on a trial $i$ is given by the formula:

$$
\Delta \mathrm{V}_{i}=\alpha \beta(\lambda-\overline{\mathrm{V}}),
$$

where $\alpha$ and $\beta$ are learning rate parameters associated with the conditioned stimulus (CS) and unconditioned stimulus (US), respectively, $\lambda$ is the maximum conditioning supportable by the US, and $\bar{V}$ is the current associative strength. Within the Rescorla-Wagner model, $\alpha$ is generally taken to refer to the salience of the CS.

We thank past students in our Associative Learning course (PSY-325) for their suggestions on ways to improve the programs. Please address correspondence to N. W. Bond, School of Behavioural Sciences, Macquarie University, Sydney, New South Wales 2109, Australia.
Each simulation of the model is preceded by text screens that describe the simulation and indicate what the students will have to key in and what they will see.

\section{Acquisition}

The first simulation involves the acquisition of associative strength. Students are invited to enter values (between 0 and 1) for alpha for each of three separate CSs, and for beta and lambda for the US. A graph is then drawn in high-resolution mode illustrating the acquisition of associative strength by each of the CSs, as if each were paired separately and independently with the US. This allows an initial comparison of the effects of alpha on the rate of acquisition of associative strength. Students can then repeat the simulation to examine the effects of changing lambda or beta.

\section{Extinction}

The simulation of extinction allows for a comparison of the shape of the acquisition curve with the shape of the extinction curve. It also allows students to examine the effects of different parameter values on extinction.

\section{Overshadowing}

A major strength of the Rescorla-Wagner model is its ability to account for the allocation of associative strength when stimuli are presented in compound. In the present simulation, a student enters values for alpha for each of two separate CSs and values for beta and lambda for a single US. The student is then shown a simulation of the acquisition of associative strength for each $\mathrm{CS}$ as if each had been separately paired with the US. This is followed by a simulation of the allocation of associative strength to each CS, and the sum of the associative strengths when the CSs are presented in compound. The simulation demonstrates that each $\mathrm{CS}$ acquires associative strength in relation to its salience (alpha).

\section{Blocking}

Blocking is said to occur when the CS is paired with a US and, as a result of the prior pairing, prevents a second CS from acquiring associative strength when both CSs are subsequently presented in compound with the US. 
Blocking is simulated in a similar manner to that used for overshadowing. The student enters values for two CSs (CSA and CSB) and a single US. The acquisition of associative strength by each CS, paired separately with the US, is then illustrated. A sample of the output is shown in the top half of Figure 1. The second simulation pairs CSA with the US for 10 trials and then presents CSA and CSB in compound, paired with the US for the remaining trials. The simulation depicts the acquisition of associative strength by each of the elements and by the compound. The initial pairing of CSA with the US allows CSA to acquire associative strength prior to the introduction of CSB. Because CSA predicts the occurrence of the US, little associative strength accrues to CSB (i.e., CSA blocks conditioning to CSB). The lower half of Figure 1 illustrates the simulation of blocking. Students can modify the rate at which associative strength accrues to CSA and thus modulate the degree to which blocking occurs.

\section{Conditioned Inhibition}

The Rescorla-Wagner model also provides an account of the acquisition of conditioned inhibition (Wagner \& Rescorla, 1972). This account treats the acquisition of conditioned inhibition as the acquisition of negative associative strength. The simulation is based on the technique
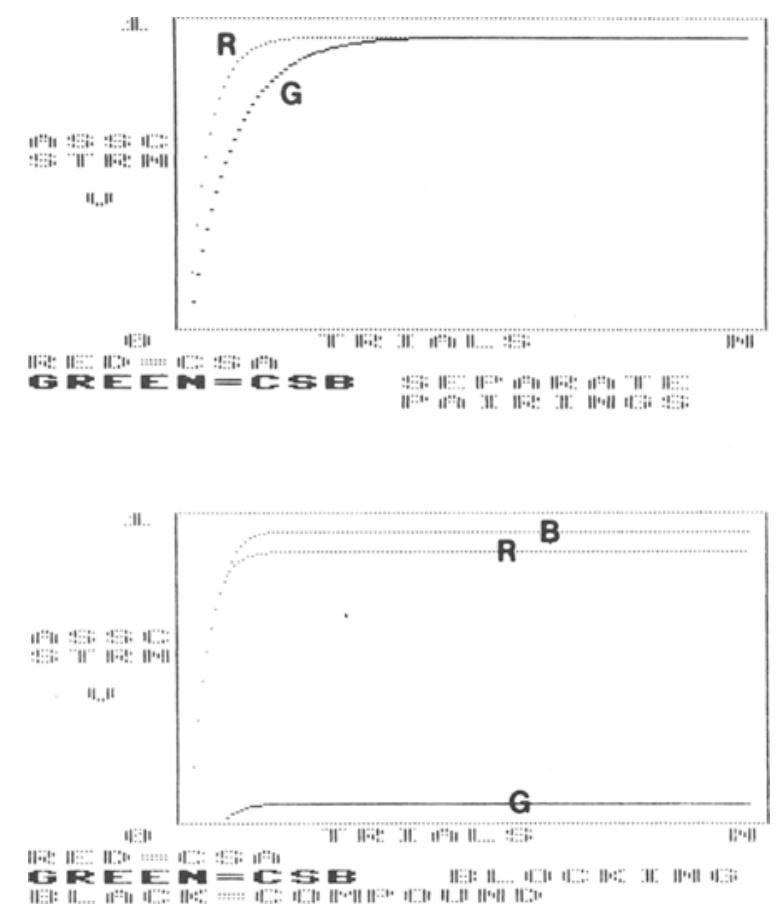

Figure 1. Top: Acquisition of associative strength (V) when conditioned stimulus A (CSA) and conditioned stimulus B (CSB) were each paired separately with the US. Bottom: Acquisition of associative strength (V) by CSA, CSB, and their compound, when CSA was paired with the US for 10 trials prior to being compounded with CSB. The compound was then paired with the US for the remaining trials. $R$ stands for RED, G for GREEN, and B for BLACK. The values for each simulation were: $\lambda=1, \alpha(\mathrm{CSA})=1, \alpha(\mathrm{CSB})$ $=.5$, and $\beta=.2$. of producing conditioned inhibition by pairing CSA alone with the US, and interspersing presentations of CSA + CSB in the absence of the US. As positive associative strength accrues to CSA following pairings with the US, so negative associative strength (conditioned inhibition) accrues to CSB, which predicts the absence of the US, even in the presence of an excitatory stimulus (CSA). The simulation illustrates the changes in the individual associative strengths of CSA and CSB, together with that of the compound.

\section{Latent Inhibition}

Because alpha is said to be a fixed-rate learning parameter, the original Rescorla-Wagner model cannot account for the effects of CS preexposure. The retardation of conditioning as a result of CS preexposure is known as latent inhibition. To account for latent inhibition, Wagner (1978) extended the original model by proposing that changes in the predictability of the CS could be thought of in much the same way as could changes in the predictability of the US. Wagner suggested that changes in associative strength can be expressed as follows:

$$
\Delta \mathrm{V}_{i}=\alpha(\gamma-\overline{\mathrm{v}}) \beta(\lambda-\overline{\mathrm{V}}),
$$

where $(\gamma-\bar{v})$ represents processing of the CS. Specifically, $\gamma$ is the maximum conditioning supportable by the $\mathrm{CS}$, and $\bar{v}$ is the current associative strength of cues that signal the occurrence of the CS. Using this equation, we can simulate latent inhibition. The CS is paired with a US, and the acquisition of associative strength is illustrated. This is then compared with the case in which the CS has previously been presented on its own, 10 times, prior to being paired with the US (i.e., latent inhibition).

\section{US Expectancy}

This simulation is based upon an experiment reported by Kremer (1978). Kremer posed the question of what would happen if, following separate pairings to asymptote of CSA and one food pellet and CSB and one food pellet, CSA and CSB were compounded and followed by only one pellet. The Rescorla-Wagner model predicts that the animal will expect two pellets and, after the animal receives only one, the associative strengths of CSA and CSB will have to decline to nullify the discrepancy between what the animal expects (i.e., two pellets) and what it actually receives (i.e., one pellet). The simulation illustrates the separate acquisition of associative strength by CSA and CSB and the loss of associative strength when CSA and CSB are compounded. It also demonstrates the counterintuitive prediction that the CS with the greatest salience loses the most associative strength.

\section{OPPONENT-PROCESS THEORY}

The opponent-process theory, proposed by Solomon and Corbit (1974), has its origins in sensory perception (see Hurvich \& Jamison, 1957). Basically, it suggests that a hedonic stimulus elicits an A state, the primary emotion 
associated with the stimulus. The $\mathrm{A}$ state then recruits an opposite state, the B state. With repeated presentations of the hedonic stimulus, the A state remains unchanged, but the B state occurs earlier, increases in magnitude, and takes longer to dissipate. As a result of the changes associated with repeated presentations of the hedonic stimulus, the primary emotion is attenuated, and, following the removal of the stimulus, the opposite emotion is experienced more strongly. If the stimulus is not presented, the changes in the B state attenuate and, as a result, the primary emotion is experienced to a greater extent. The present program utilizes the example provided by Hurvich and Jamison (1957) in which a red screen is followed by a gray screen, a procedure that illustrates the changes in saturation and in the afterimage.

To simulate the Solomon-Corbit model, the program utilizes parachute jumping as the hedonic stimulus. The changes in the A state, the B state, and their sum (the manifest response) are presented graphically (see Solomon \& Corbit, 1974, Figure 4A). If a "jump" occurs within $10 \mathrm{sec}$ of the previous jump, the B state increments, occurring with a shorter latency and greater magnitude, and taking longer to decay. A sequence of jumps, each occurring within the 10-sec limit, leads to an increase in the B state to the point that it almost cancels out the A state (see Solomon \& Corbit, 1974, Figure 4B). It is worth noting that the Solomon and Corbit model is not explicit about the changes in the form of the B state. However, using their Figure 4 as a guide, the changes can be simulated using a linear-operator model similar to that of Rescorla and Wagner. If the student refrains from jumping for a period of time, the $B$ state will decline. As each 10-sec period elapses, the B state decays, until it reverts to its original level. By jumping or not jumping, the student can modulate changes in the B state and, thus, in the manifest response, as predicted by the Solomon-Corbit model.

\section{CONDITIONED OPPONENT-PROCESS THEORY}

Schull (1979) has modified the Solomon-Corbit opponent-process theory by suggesting that there are two B states. One is recruited by the A state and does not change following repeated presentations. Schull has suggested that changes in the second type of B state result from conditioning. Stimuli (CSs) that predict the hedonic stimulus (which can be viewed as a US) come to elicit a conditioned response, the B state, which is opposite in direction to the A state and which attenuates its effects. This modification means that changes in the B state are not brought about by presentations of the hedonic stimulus per se, but by presentation of the cues that predict the hedonic stimulus.

The example employed is based on Siegel's (1975) work on tolerance to the analgesic effects of morphine. The stu- dent can present the context (CS) in association with the drug (US). Changes in the A state, in both the invariant and the conditioned B states, and in their sum (the manifest response) are displayed graphically (see Schull, 1979, Figure 2). Repeated pairings of the context and the drug lead to increases in the conditioned B state, to the point at which, together with the invariant B state, it almost cancels out the effects of the A state (see Schull, 1979, Figure 2). Schull is explicit in drawing together opponent-process theory and the Rescorla-Wagner model, and the changes in the B state are based upon the latter. If the student refrains from presenting the context or the drug, there is no change in the conditioned B state, notwithstanding the passage of time. However, if the student presents the context in the absence of the drug, the conditioned B state declines (i.e., extinction occurs). Repeated presentations of the context alone lead to complete extinction of the conditioned $B$ state. The simulation demonstrates that the changes in the $B$ state are not due to the interval between presentations of the hedonic stimulus, but are due instead to presentations of the contextual stimuli that predict the hedonic stimulus.

\section{AVAILABILITY}

Instructions and copies of the programs on disk, formatted for the 1541 disk drive or compatibles, may be obtained for the cost of copying and mailing (U.S. \$15).

\section{REFERENCES}

HuRvich, L. M., \& Jamison, D. (1957). An opponent-process theory of color vision. Psychological Review, 64, 384-404.

Kremer, E. F. (1978). The Rescorla-Wagner model: Losses in associative strength in compound conditioned stimuli. Joumal of Experimental Psychology: Animal Behavior Processes, 4, 22-36.

Rescorla, R. A., \& Wagner, A. R. (1972). A theory of Pavlovian conditioning: Variations in the effectiveness of reinforcement and nonreinforcement. In A. H. Black \& W. F. Prokasy (Eds.), Classical conditioning 2. New York: Appleton-Century-Crofts.

Schull, J. A. (1979). A conditioned-opponent process theory of Pavlovian conditioning and habituation. In G. H. Bower (Ed.), The psychology of learning and motivation (Vol. 13). New York: Academic Press.

SIEGEL, S. (1975). Evidence from rats that morphine tolerance is a leamed response. Joumal of Comparative \& Physiological Psychology, 89, 498-506.

Solomon, R. L., \& CoRBIT, J. D. (1974). An opponent-process theory of motivation: 1. Temporal dynamics of affect. Psychological Review, 81, 119-145.

WAGNER, A. R. (1978). Expectancies and the priming of STM. In S. H. Hulse, H. Fowler, \& W. K. Honig (Eds.), Cognitive processes in animal behavior. Hillsdale, $\mathrm{NJ}$ : Erlbaum.

Wagner, A. R., Rescorla, R. A. (1972). Inhibition in Pavlovian conditioning: Applications of a theory. In R. A. Boakes \& M. S. Halliday (Eds.), Inhibition and leaming. New York: Academic Press.

(Manuscript received July 17, 1986; revision accepted for publication April 16, 1987.) 